\title{
The Construction of Talents Cultivating Plan Based on Hotel Management Major of Systematic Working Process
}

\author{
Quan-Jun Zheng, You-Rong Xiao \\ Hainan College of Software Technology, QiongHai, Hainan 571400, China \\ Zhengqj67@126.com Xiaoyor69@126.com
}

\begin{abstract}
The development of curriculum system based on systematic working process follows the process of "Post (group) investigation analysis and research (including processional growth phase nanlysis ) $\rightarrow$ work task decomposition $\rightarrow$ action in induction $\rightarrow$ learning areas conversion $\rightarrow$ learning situation design". This thesis constructs hotel management major talents cultivating plan according to development rule of systematic work process of curriculum system.

Index Terms - Hotel Management Major, Systematic working process, Talents cultivating plan
\end{abstract}

\section{Introduction}

The step of curriculum system development based on systematic working process is simply expounded as "Post (group) investigation analysis and research(including processional growth phase nanlysis ) $\rightarrow$ work task decomposition $\rightarrow$ action in induction $\rightarrow$ learning areas conversion $\rightarrow$ learning situation design", like figure1[1]:

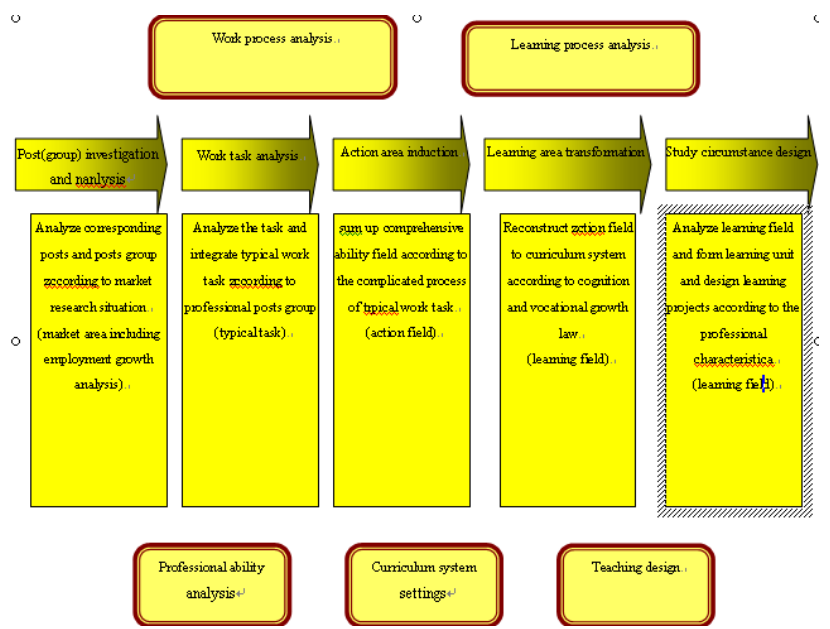

Figure1: The curriculum system development flowchart based on systematic work process

\section{The Talents Cultivating Plan Based on Hotel Management Major of Systematic Working Process}

\subsection{The recruiting students - academic structure and credit of} hotel management major

The recruiting students: high enrollment of national test, the qualificator of examination awards college diploma of education of the people's republic of china. Academic structure: 3 years. The credit of graduation: 177.5(including top post practice and graduation project: 36 credits, awarding credits: 6 credits, moral training: 1.5 credits)

\subsection{The training target of hotel management major}

The training target is to cultivate high-quality and high skill applied management expertise who owns good moral, proper etiquette and can fully master basic theory of modern hotel management and be faniliar with basic skill of hotel service and hotel management.

\subsection{The jobs of hotel management major}

The jobs (group) of hotel management graduates are hotel service post, management skill post and bar management post. And the management skill post is departed to front office management post, food and bevetage management post and guest room management post etc. The developed expanding posts are dieticians, bartenders, convention and exhibitionservices and other related jobs.

\subsection{The vocational skills certificate request of hotel management major}

Before graduation the hotel management graduate should get: (1) English proficiency certificate; (2) Computer certificates; (3) Professional skill certificate; (4) Job qualification certificate. And the students must get one professional skill certificate at least. The name of certificate, issuing agency and courses are in chart1:

Chart1: The vocational skill certificates list

\begin{tabular}{|l|l|l|}
\hline Certificate Name & Issuing agency & Courses \\
\hline $\begin{array}{c}\text { 1. National certificate of college english application ability of A- } \\
\text { level and above or the national level and above IT vocational }\end{array}$ & College english proficiency test commission & College English \\
$\begin{array}{l}\text { english proficiency test certificate or the national english test } \\
\text { certificate of three-level and above }\end{array}$ & $\begin{array}{l}\text { Electronic education and examination center of } \\
\text { the ministry of information industry }\end{array}$ & Computer Application Foundation \\
2. National computer test certificate of one-level and above & NEEA & Restaurant service and \\
management $\cdot$ Guest room service \\
3. Professional skill certificate and Job qualification certificate & NEEA & and management Recreation \\
(1)Hotel management professional & The highes and management etc. \\
(2)Catering certificates of professional managers & committee and the China Cuisine Association & Foodservice and management etc. \\
\end{tabular}




\subsection{The overall arrangement of all teaching term for hotel management major(like chart2)}

Chart 2: The overall arrangement of all teaching term

\begin{tabular}{|c|c|c|c|c|c|c|c|c|c|}
\hline \multirow{2}{*}{$\begin{array}{l}\text { School } \\
\text { year }\end{array}$} & \multirow{2}{*}{ term } & \multirow{2}{*}{$\begin{array}{l}\text { Teaching } \\
\text { weeks }\end{array}$} & \multicolumn{4}{|c|}{ Centralized Practice (week) } & \multirow{2}{*}{$\begin{array}{l}\text { Review and exam } \\
\text { (week) }\end{array}$} & \multirow{2}{*}{$\begin{array}{l}\text { Total weeks } \\
\text { (week) }\end{array}$} & \multirow{2}{*}{ Comment } \\
\hline & & & $\begin{array}{l}\text { Military } \\
\text { training }\end{array}$ & $\begin{array}{l}\text { Mukti-skilled } \\
\text { training }\end{array}$ & $\begin{array}{l}\text { Top post } \\
\text { training }\end{array}$ & $\begin{array}{c}\text { Graduated } \\
\text { design }\end{array}$ & & & \\
\hline \multirow{3}{*}{1} & 1 & 15 & 2 & 1 & & & 1 & 19 & One week later \\
\hline & 2 & 16 & & 2 & & & 2 & 20 & \\
\hline & 3 & & & 4 & & & & 4 & \\
\hline \multirow{3}{*}{2} & 1 & 16 & & 2 & & & 2 & 20 & \\
\hline & 2 & 16 & & 2 & & & 2 & 20 & \\
\hline & 3 & & & 4 & & & & 4 & \\
\hline \multirow[t]{2}{*}{3} & 1 & & & & 20 & & & 20 & \\
\hline & 2 & & & & 8 & 8 & & 16 & $\begin{array}{c}\text { Last semester lack } \\
4 \text { weeks }\end{array}$ \\
\hline \multicolumn{2}{|c|}{ Total } & 63 & 2 & 15 & 28 & 8 & 7 & 123 & \\
\hline
\end{tabular}

\subsection{The development process of hotel management major curriculum system(like chart3)}

Chart3: The development process of hotel management major curriculum system

\begin{tabular}{|c|c|c|c|c|c|}
\hline \multicolumn{2}{|c|}{ Post name } & Typical work task & Action field & Learning field & $\begin{array}{l}\text { Career } \\
\text { development }\end{array}$ \\
\hline \multicolumn{2}{|c|}{ Hotel service post } & $\begin{array}{l}\text { service work of all } \\
\text { post in hotel sectors }\end{array}$ & $\begin{array}{l}\text { front office service, housekeeping service, } \\
\text { catering service, conference service, } \\
\text { recreation and sports service, bar service and } \\
\text { beverage deployment }\end{array}$ & $\begin{array}{l}\text { Psychology of hotel service, Hotel } \\
\text { manners, hotel service, Recreation } \\
\text { servicesand management, Bar } \\
\text { service }\end{array}$ & Work ability \\
\hline \multirow{3}{*}{$\begin{array}{l}\text { Hotel } \\
\text { Management } \\
\text { technical } \\
\text { posts }\end{array}$} & $\begin{array}{l}\text { Guest room } \\
\text { managemen } \\
\text { t post }\end{array}$ & $\begin{array}{l}\text { Housekeeping } \\
\text { management and } \\
\text { day-to-day } \\
\text { management work }\end{array}$ & $\begin{array}{l}\text { guest room design and environmental } \\
\text { management, quality control of guest rooms, } \\
\text { rooms supplies management, room equipment } \\
\text { management, rooms safety management, room } \\
\text { budget management, rooms business planning } \\
\text { and reconstruction, room information } \\
\text { management and personnel management etc. }\end{array}$ & $\begin{array}{l}\text { Guest room service and } \\
\text { management, hotel public, hotel } \\
\text { human resources management }\end{array}$ & \multirow{3}{*}{$\begin{array}{l}\text { Job-hunting } \\
\text { ability }\end{array}$} \\
\hline & $\begin{array}{l}\text { Front office } \\
\text { managemen } \\
\text { t post }\end{array}$ & $\begin{array}{l}\text { Front office } \\
\text { management and } \\
\text { day-to-day } \\
\text { management work }\end{array}$ & $\begin{array}{l}\text { guest room price management, quality control, } \\
\text { sales management, front officel information } \\
\text { management and personnel management etc }\end{array}$ & $\begin{array}{l}\text { Front Office service and } \\
\text { management, customer service and } \\
\text { management, hotel public } \\
\text { relations, hotel human resources } \\
\text { management, hotel finance }\end{array}$ & \\
\hline & $\begin{array}{l}\text { Food and } \\
\text { beverage } \\
\text { managemen } \\
t \text { post }\end{array}$ & $\begin{array}{l}\text { Food and beverage } \\
\text { management and } \\
\text { day-to-day } \\
\text { management work }\end{array}$ & $\begin{array}{l}\text { raw materials management, production } \\
\text { management, service management, sales } \\
\text { management, financial management, catering } \\
\text { information management and personnel } \\
\text { management etc. }\end{array}$ & $\begin{array}{l}\text { Food and beverage service and } \\
\text { management, food nutrition and } \\
\text { health, hotel public relations, hotel } \\
\text { human resources management, } \\
\text { hotel finance }\end{array}$ & \\
\hline \multicolumn{2}{|c|}{ Bar management posts } & $\begin{array}{l}\text { Bar operation and } \\
\text { management }\end{array}$ & $\begin{array}{l}\text { Business planning; registration; business } \\
\text { management }\end{array}$ & Bar management & $\begin{array}{l}\text { Creative } \\
\text { ability }\end{array}$ \\
\hline
\end{tabular}




\subsection{The curriculum planning table of hotel management major(like chart4)}

Chart4: The curriculum planning table of hotel management major

\begin{tabular}{|c|c|c|c|c|c|c|c|c|c|c|c|c|c|c|}
\hline \multirow{2}{*}{ courses } & \multirow{2}{*}{\multicolumn{2}{|c|}{ Course name }} & \multirow{2}{*}{$\begin{array}{l}\text { Total } \\
\text { credits }\end{array}$} & \multirow{2}{*}{$\begin{array}{l}\text { Theory } \\
\text { class }\end{array}$} & \multirow{2}{*}{$\begin{array}{c}\text { Practice } \\
\text { class }\end{array}$} & \multicolumn{3}{|c|}{ First year } & \multicolumn{3}{|c|}{ Second year } & \multicolumn{2}{|c|}{ Third year } & \multirow[t]{2}{*}{ notes } \\
\hline & & & & & & 一 & 二 & 三 & 一 & 二 & 三 & 一 & 二 & \\
\hline \multirow{9}{*}{$\begin{array}{l}\text { Public } \\
\text { course }\end{array}$} & \multicolumn{2}{|c|}{ Basis of morality accomplishment and law } & 3 & 3 & & 3 & & & & & & & & \\
\hline & \multicolumn{2}{|l|}{ College english } & 8 & 8 & & $4 *$ & 4 & & & & & & & \\
\hline & \multicolumn{2}{|c|}{ Computer application foundation } & 4 & 2 & 2 & $4 *$ & & & & & & & & \\
\hline & \multicolumn{2}{|l|}{ College P.E } & 2 & 2 & & 2 & 2 & & & & & & & \\
\hline & \multicolumn{2}{|l|}{ Mental health } & 2 & 2 & & & & & & & & & & lecture \\
\hline & \multicolumn{2}{|l|}{ Military theory } & 2 & 2 & & & & & & & & & & lecture \\
\hline & \multicolumn{2}{|c|}{ Situation and policies } & 4 & 3 & 1 & & & & & & & & & lecture \\
\hline & \multicolumn{2}{|c|}{ Career development and employment guidance } & 2 & 2 & & & & & & & & & & lecture \\
\hline & \multicolumn{2}{|c|}{ Military training } & 2 & & 2 & & & & & & & & & $\begin{array}{c}\text { concentrate } \\
\text { d study }\end{array}$ \\
\hline \multicolumn{3}{|l|}{ Total } & 29 & 24 & 5 & 13 & 6 & & & & & & & \\
\hline \multirow{8}{*}{$\begin{array}{c}\text { Post } \\
\text { basic } \\
\text { course }\end{array}$} & \multicolumn{2}{|c|}{ Travel writing and eloquence } & 4 & 4 & & 2 & $2 *$ & & & & & & & \\
\hline & \multicolumn{2}{|c|}{ Hotel management introduction } & 4 & 4 & & $4^{*}$ & & & & & & & & \\
\hline & \multicolumn{2}{|c|}{ Psychology of hotel service } & 2 & 2 & & 2 & & & & & & & & \\
\hline & Hotel financia & 1 management & 4 & 2 & 2 & & & & $4^{*}$ & & & & & \\
\hline & Hotel engliah & & 4 & 2 & 2 & & & & $4^{*}$ & & & & & \\
\hline & Hotel public $r$ & elation & 4 & 2 & 2 & & $4 *$ & & & & & & & \\
\hline & Hotel manners & & 5 & 2 & 3 & $4 *$ & & & & & & & & $\begin{array}{c}\text { Practice } \\
\text { one week }\end{array}$ \\
\hline & Body training & & 4 & & 4 & 1 & 1 & & 1 & 1 & & & & \\
\hline & Hotel service & & 5 & 2 & 3 & & $4 *$ & & & & & & & $\begin{array}{l}\text { Practice } \\
\text { one week }\end{array}$ \\
\hline & Front office se & rvice and management & 6 & 3 & 3 & & & & & $6^{*}$ & & & & \\
\hline & Food service a & nd management & 7 & 3 & 4 & & $6^{*}$ & & & & & & & $\begin{array}{c}\text { Practice } \\
\text { one week }\end{array}$ \\
\hline & Beverage servi & ice and bar management & 7 & 3 & 4 & & & & $6^{*}$ & & & & & $\begin{array}{c}\text { Practice } \\
\text { one week }\end{array}$ \\
\hline Job skill & Room service & and management & 9 & 4 & 5 & & & & 4 & $4 *$ & & & & $\begin{array}{c}\text { Practice } \\
\text { one week }\end{array}$ \\
\hline course & Human resour & ces management in hotel & ----7 & 3 & 4 & & & & & $6^{*}$ & & & & $\begin{array}{c}\text { Practice } \\
\text { one week }\end{array}$ \\
\hline & Recreation ser & vice and management & 7 & 3 & 4 & & & & & $6^{*}$ & & & & $\begin{array}{c}\text { Practice } \\
\text { one week }\end{array}$ \\
\hline & Food nutrition & and sanitation & 4 & 2 & 2 & & & & $4^{*}$ & & & & & \\
\hline & Hotel service $t$ & raining & 4 & & 4 & & & 30 & & & & & & \\
\hline & Hotel manager & ment training & 4 & & 4 & & & & & & 30 & & & \\
\hline & Post practice a & and graduated design & 36 & & 36 & & & & & & & 30 & 30 & \\
\hline & & total & 127 & 41 & 86 & 13 & 17 & 30 & 23 & 23 & 30 & 30 & 30 & \\
\hline $\begin{array}{c}\text { Post } \\
\text { expandin }\end{array}$ & $\begin{array}{l}\text { Specialized } \\
\text { Choice }\end{array}$ & $\begin{array}{l}\text { Liquor - blending knowledge } \\
\text { and liquor - blending, the party } \\
\text { design, training of professional } \\
\text { order, Cooking and Food }\end{array}$ & 8 & 4 & 4 & & & & & & & 8 & & $\begin{array}{c}\text { Choose } 2 \\
\text { from } 4\end{array}$ \\
\hline $\mathrm{g}$ course & Public course & total 6 credits & 6 & 6 & & & 2 & & 2 & 2 & & & & \\
\hline & & total & 14 & 10 & 4 & & 2 & & 2 & 2 & & 8 & & \\
\hline & & total & 170 & 75 & 95 & 26 & 25 & 30 & 25 & 25 & 30 & 38 & 30 & \\
\hline
\end{tabular}

Note: $*$ denotes the course are examination subjectthis term 


\subsection{The learning circumstance design of hotel management \\ major}

Here setting the course-FoodService and Management as

example to simply prove the learning ciecumstance design

based on systematic work process, like chart 5:

Chart5: Learning circumstance design chart of FoodService and Management course

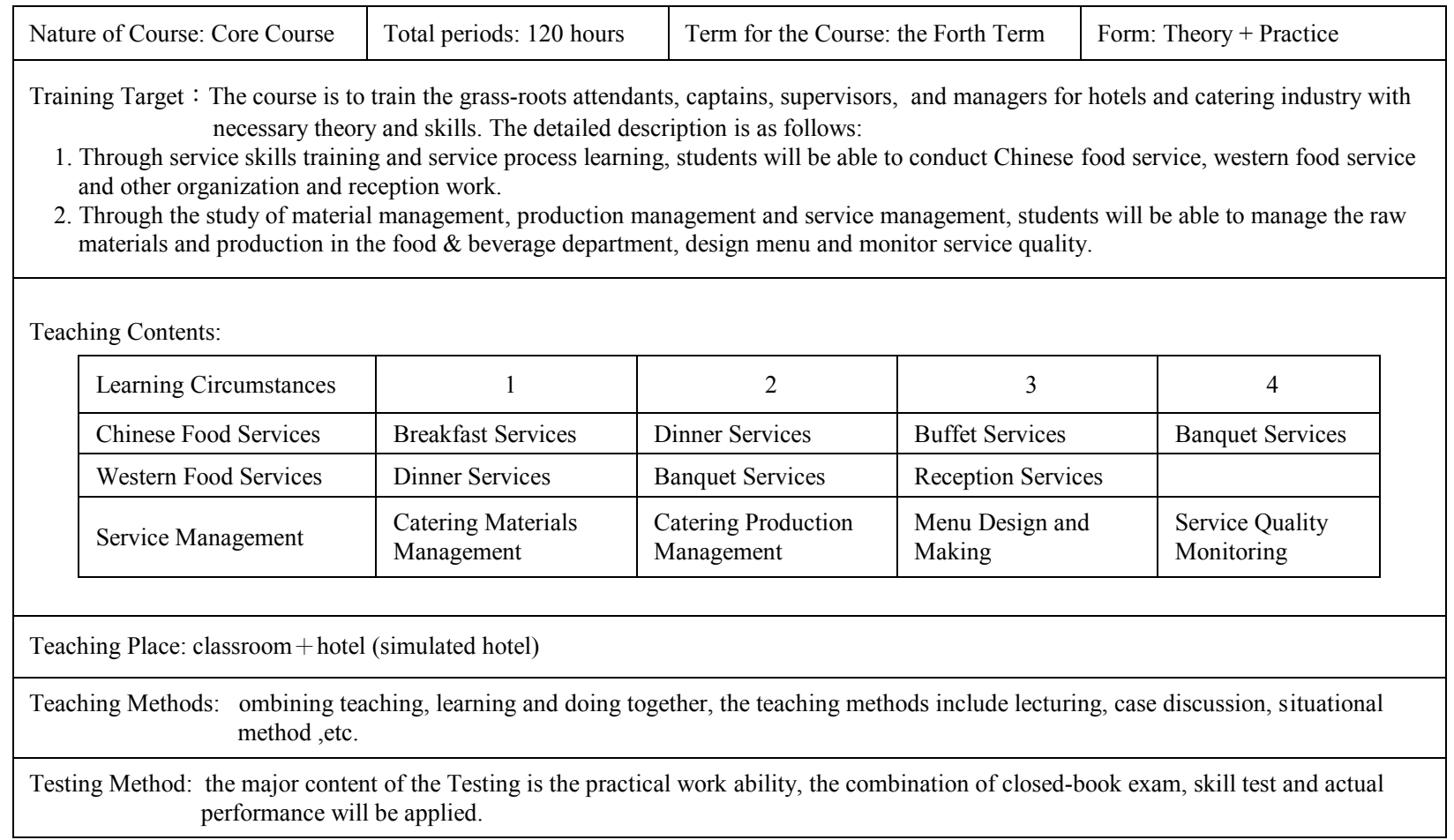

\section{Acknowledgment}

This thesis has received the support from the project of 2013 Hainan Psychology and Sociology Planning (HNSK (GJ) 13-122) and 2013 Teaching Reform for Hainan Vocational Education (Hngjy13-029) . Thanks a lot!

\section{References}

[1] Zhan-Ming Liu, Course System Innovation Research Based on Work Process-oriented Higher Vocational College Tourism. Hebei Normal University. 2010(02):28.

[2] Tong-He Shi, The Development and Reconstruction of Course System Based on work process-oriented Higher Vocational College Hotel Management major, China Electronic Education,. 2009 (07):141. 